\title{
A PURELY SYMBOL-BASED PRECODED AND LDPC-CODED ITERATIVE-DETECTION ASSISTED SPHERE-PACKING MODULATED SPACE-TIME CODING SCHEME
}

\author{
O. Alamri, S. X. Ng, F. Guo and L. Hanzo* \\ School of ECS, University of Southampton, SO17 1BJ, UK. \\ Email: 1h@ecs.soton.ac.uk \\ http://www-mobile.ecs.soton.ac.uk
}

\begin{abstract}
In this contribution, we propose a purely symbolbased LDPC-coded scheme based on a Space-Time Block Coding (STBC) signal construction method that combines orthogonal design with sphere packing, referred to here as (STBCSP). We demonstrate that useful performance improvements may be attained when sphere packing aided modulation is concatenated with non-binary LDPC especially, when performing purely symbol-based turbo detection by exchanging extrinsic information between the non-binary LDPC decoder and a rate-1 non-binary inner precoder. We also investigate the convergence behaviour of this symbol-based concatenated scheme with the aid of novel non-binary Extrinsic Information Transfer (EXIT) Charts. The proposed symbol-based turbo-detected STBC-SP scheme exhibits a 'turbo-cliff' at $E_{b} / N_{0}=5.0 \mathrm{~d} B$ and achieves an $E_{b} / N_{0}$ gain of $19.2 d B$ at a $B E R$ of $10^{-5}$ over Alamouti's scheme.
\end{abstract}

\section{INTRODUCTION}

The benefits of transmit diversity may be exploited by employing space-time coding invoking multiple antennas both at the transmitter and the receiver $[1,2]$. A simple yet efficient transmit diversity scheme employing two transmit antennas was discovered by Alamouti [3]. The low complexity of Alamouti's design further inspired Tarokh et al. [4] to generalise the transmit diversity scheme of [3] to an arbitrary number of transmit antennas using the principle of orthogonal design. Since then, the pursuit of designing better space-time modulation schemes has attracted considerable further attention [2]. The concept of combining orthogonal transmit diversity designs with the principle of sphere packing was introduced by Su et al. in [5], where it was demonstrated that the proposed Sphere Packing (SP) aided Space-Time Block Coded (STBC) system, referred to here as STBC-SP, was capable of outperforming the conventional orthogonal design based STBC schemes of $[3,4]$.

Low Density Parity Check (LDPC) codes originally devised by Gallager as early as 1963 [6] have experienced a renaissance [7] and attracted substantial research interests since the discovery of turbo codes in 1993 [8]. In 1998, Davey and MacKay proposed a non-binary version of LDPC codes [9], which was potentially capable of outperforming their binary counterpart [6].

Iterative decoding of spectrally efficient modulation schemes was considered by several authors. In [10], the employment of the turbo principle was considered for iterative soft demapping in the context of multilevel modulation schemes combined with channel decoding, where a soft demapper was used between the

*The financial support of the European Union under the auspices of the Newcom and Phoenix projects, as well as that of the EPSRC UK and the Ministry of Higher Education of Saudi Arabia is gratefully acknowledged. multilevel demodulator and the channel decoder. In [11, 12], rate1 inner codes were employed for designing low complexity turbo codes suitable for bandwidth and power limited systems having stringent bit-error-rate $(B E R)$ requirements.

Recently, studying the convergence behaviour of iterative decoding has attracted considerable attention. In [13], ten Brink proposed the employment of the so-called extrinsic information transfer (EXIT) characteristics between a concatenated decoder's output and input for describing the flow of extrinsic information through the soft in/soft out constituent decoders. EXIT charts have been extended to the non-binary (index-based) case in [14], where a histogram-based approximation of the extrinsic information was required in order to compute the mutual information. An efficient and low-complexity method of computing non-binary EXIT charts from index-based a posteriori probabilities was proposed in [15], which is considered a generalisation of the approach presented in [16].

Motivated by the performance improvements reported in [5], [9] and [11,12], we propose a novel purely symbol-based iterative system. Given a certain effective throughput, our simulation results demonstrate that the proposed non-binary turbo-detection aided STBC-SP scheme is capable of providing attractive performance improvements over established orthogonal STBC designs, constituted by the STBC-SP scheme of [5] as well as over a binary LDPC-coded turbo-detected STBC-SP benchmarker scheme. Explicitly, as a benefit of the proposed solution, it will be demonstrated in Section 5 that the proposed turbo-detection aided STBCSP scheme is capable of providing an $E_{b} / N_{0}$ gain of $19.2 d B$ at a Bit Error Rate $(B E R)$ of $10^{-5}$ over the STBC-SP scheme of [5]. The novel non-binary EXIT charts of [14] are employed for designing our non-binary scheme.

This paper is organised as follows. A system overview is presented in Section 2. Symbol-based iterative decoding is described in Section 3. Section 4 provides our EXIT chart analysis, while our simulation results are discussed in Section 5. Finally, we conclude in Section 6

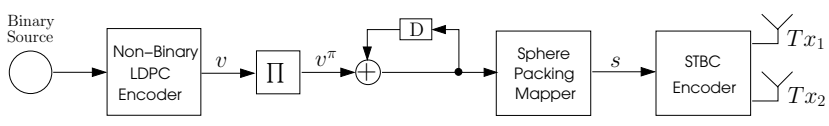

Figure 1: Symbol-Based Turbo Detection STBC-SP Encoder.

\section{SYSTEM OVERVIEW}

In this contribution, space-time systems employing two transmit antennas are considered, where the space-time signal is given by [3]

$$
G_{2}\left(x_{1}, x_{2}\right)=\left[\begin{array}{cc}
x_{1} & x_{2} \\
-x_{2}^{*} & x_{1}^{*}
\end{array}\right] \text {, }
$$




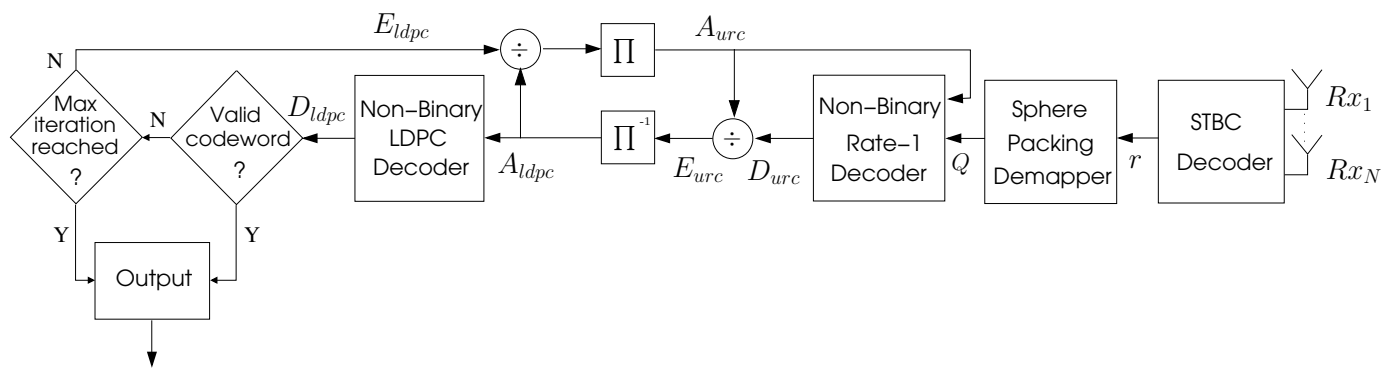

Figure 2: Symbol-Based Turbo Detection STBC-SP Decoder.

and the rows as well as columns of Equation (1) represent the temporal and spatial dimensions, corresponding to two consecutive time slots and two transmit antennas, respectively. The schematic of the proposed turbo-detected purely symbol-based scheme is shown in Figure 1 . The source bits are encoded by a rate $R=\frac{1}{2}$ nonbinary LDPC encoder [9], to generate the LDPC encoded symbols $\mathbf{v}=\left\{v_{0}, v_{1}, \ldots, v_{K-1}\right\}, v_{k} \in G F(q)$, where $K$ is the LDPC output block length and $q$ is the size of the LDPC decoding field. The LDPC encoded symbols are interleaved and then precoded by a non-binary rate- 1 encoder, before each of them is mapped to the corresponding sphere packing modulated symbol $s^{l} \in S$, $0 \leq l \leq L-1$. More specifically, $S=\left\{s^{l}=\left[a_{l, 1}, a_{l, 2}, a_{l, 3}, a_{l, 4}\right]\right.$ $\left.\in \mathrm{R}^{4}: 0 \leq l \leq L-1\right\}$ constitutes a set of $L$ legitimate constellation points selected from the lattice $D_{4}$ having a total energy of $E \triangleq \sum_{l=0}^{L-1}\left(\left|a_{l, 1}\right|^{2}+\left|a_{l, 2}\right|^{2}+\left|a_{l, 3}\right|^{2}+\left|a_{l, 4}\right|^{2}\right)$. There is a natural one-to-one mapping between $l$ and the elements of the non-binary LDPC code defined over $G F(q)$, where we have $L=q$, allowing us to create a purely symbol-based system. Again, the rate- 1 precoder shown in Figure 1 is also a non-binary encoder, defined by the binary generator polynomial $G=\left(g / g_{r}\right)=(10 / 11)$, where $g$ denotes the feedforward output and $g_{r}$ is the feedback to the input using a modulo $q$ addition. The STBC encoder then maps each sphere packing modulated symbol $s^{l}$ to a space-time signal $C_{l}=\sqrt{\frac{2 L}{E}} G_{2}\left(x_{l, 1}, x_{l, 2}\right), 0 \leq l \leq L-1$, such that $x_{l, 1}$ and $x_{l, 2}$ are written as

$$
\begin{aligned}
\left\{x_{l, 1}, x_{l, 2}\right\} & =T\left(a_{l, 1}, a_{l, 2}, a_{l, 3}, a_{l, 4}\right) \\
& =\left\{a_{l, 1}+j a_{l, 2}, a_{l, 3}+j a_{l, 4}\right\} .
\end{aligned}
$$

Subsequently, each sphere-packed space-time coded symbol is transmitted over $T=2$ consecutive time slots using two transmit antennas, as shown in Equation (1). More detailed discussions on orthogonal design with sphere packing modulation are provided in [17].

In this treatise, we considered a correlated narrowband Rayleigh fading channel, associated with a worst-case-type normalised Doppler frequency of $f_{D}=f_{d} T_{s}=0.01$, where $f_{d}$ is the Doppler frequency and $T_{s}$ is the symbol duration. The complex fading envelope is assumed to be constant across the transmission period of a sphere-packed space-time coded symbol, spanning $T=2$ time slots. The complex Additive White Gaussian Noise (AWGN) of $n=n_{I}+j n_{Q}$ is also added to the received signal, where $n_{I}$ and $n_{Q}$ are two independent zero mean Gaussian random variables having a variance of $\sigma_{n}^{2}=\sigma_{n_{I}}^{2}=\sigma_{n_{Q}}^{2}=N_{0} / 2$ per dimension, with $N_{0} / 2$ representing the double-sided noise power spectral density expressed in $W / H z$.

As shown in Figure 2, the received complex-valued symbols are first decoded by the STBC decoder to produce a received spherepacked symbol $r$, which is fed into the sphere packing demapper, where the soft-metric $Q(k)$ is calculated. More explicitly, the notation $Q(k)$ represents the soft metric passed from the sphere packing demapper to the non-binary rate-1 decoder based on the probability of the $k^{t h}$ symbol of the encoded codeword by the rate- 1 en- coder. As seen in Figure 2, the rate-1 decoder processes these softmetrics in conjunction with the a-priori information, $A_{u r c}$, in order to generate the a-posteriori probability, $D_{u r c}$, where the subscript urc refers to the unity-rate code. More specifically, the a-priori information, $A_{u r c}$, is provided by the LDPC decoder as the soft metric for the LDPC encoded symbols. After removing the a-priori information, $A_{u r c}$, from the a-posteriori probability denoted by $D_{\text {urc }}$ using symbol-based element-wise division, as will be shown in Section 3, $A_{l d p c}$ is passed as a-priori information to the LDPC decoder, which carries out a specified number of LDPC iterations and produces the decoded a-posteriori probability $D_{l d p c}$. Based on the a-posteriori probability, a tentative hard decision will be made and the resultant codeword will be checked by the LDPC code's parity check matrix. If the resultant vector is an all-zero sequence, then a legitimate codeword has been found, and the hard-decision based sequence will be output. Otherwise, if the maximum affordable number of iterations has not been reached, the a-priori information, $A_{l d p c}$, is removed from the a-posteriori probability denoted by $D_{l d p c}$ using symbolbased element-wise division and fed back to the non-binary rate-1 decoder for the next iteration after appropriately reordering them using the interleaver, as seen in Figure 2. This process continues, until the affordable maximum number of iterations has been encountered or a legitimate codeword has been found.

The structure of the binary benchmarker scheme is identical to the scheme seen in Figure 1 and Figure 2, except that the rate-1 inner code is not employed and hence iterative decoding is carried out between the outer binary LDPC code and the inner bit-based sphere packing demapper over $G F(2)$.

\section{SYMBOL-BASED ITERATIVE DECODING}

For the sake of simplicity, a system having a single receive antenna is considered, although its extension to systems having more than one receive antenna is straightforward. Assuming perfect channel estimation, the complex-valued channel output symbols received during two consecutive time slots are first diversity-combined in order to extract the estimates $\tilde{x}_{1}$ and $\tilde{x}_{2}$ of the most likely transmitted symbols $x_{l, 1}$ and $x_{l, 2}$ [3][1, pp.400 - 401], resulting in

$$
\begin{aligned}
& \tilde{x}_{1}=\left(\left|h_{1}\right|^{2}+\left|h_{2}\right|^{2}\right) \cdot x_{l, 1}+\dot{w}_{1} \\
& \tilde{x}_{2}=\left(\left|h_{1}\right|^{2}+\left|h_{2}\right|^{2}\right) \cdot x_{l, 2}+\dot{w}_{2},
\end{aligned}
$$

where $h_{1}$ and $h_{2}$ represent the Channel Impulse Response (CIR) corresponding to the first and second transmit antennas, respectively, and $w_{1}$ as well as $w_{2}$ are zero-mean complex Gaussian random variables with variance $\sigma_{w_{1}}^{2}=\sigma_{w_{2}}^{2}=\left(\left|h_{1}\right|^{2}+\left|h_{2}\right|^{2}\right) \cdot \sigma_{n}^{2}$. A received sphere-packed symbol $r$ is then constructed from the estimates $\tilde{x}_{1}$ and $\tilde{x}_{2}$ using Equation (2) as $r=T^{-1}\left(\tilde{x}_{1}, \tilde{x}_{2}\right)$, where $r=\left\{\left[\tilde{a}_{1}, \tilde{a_{2}}, \tilde{a_{3}}, \tilde{a_{4}}\right] \in R^{4}\right\}$. The received sphere-packed symbol $r$ can be written as

$$
r=h \cdot \sqrt{\frac{2 L}{E}} \cdot s^{l}+w
$$


where $h=\left(\left|h_{1}\right|^{2}+\left|h_{2}\right|^{2}\right), s^{l} \in S, 0 \leq l \leq L-1$, and $w$ is a fourdimensional Gaussian random variable having a variance of $\sigma_{w}^{2}=$ $\sigma_{w}^{2}=h \cdot \sigma_{n}^{2}$, since the symbol constellation $S$ is four-dimensional. According to Equation (5), the conditional PDF $P\left(r \mid s^{l}\right)$ is given by

$$
\begin{aligned}
P\left(r \mid s^{l}\right) & =\frac{1}{\left(2 \pi \sigma_{w}^{2}\right)^{\frac{N_{D}}{2}}} e^{-\frac{1}{2 \sigma_{w}^{2}}\left(r-\alpha \cdot s^{l}\right)^{2}} \\
& =\frac{1}{\left(2 \pi \sigma_{w}^{2}\right)^{\frac{N_{D}}{2}}} e^{-\frac{1}{2 \sigma_{w}^{2}}\left(\sum_{i=1}^{4}\left(\tilde{a}_{i}-\alpha \cdot a_{l, i}\right)^{2}\right)},
\end{aligned}
$$

where we have $\alpha=h \cdot \sqrt{\frac{2 L}{E}}$ and $N_{D}=4$, since a four-dimensional sphere-packed symbol constellation is used.

Similarly, the conditional PDF $P\left(s^{l} \mid r\right)$ is given by

$$
P\left(s^{l} \mid r\right)=\frac{P\left(r \mid s^{l}\right) \cdot P\left(s^{l}\right)}{P(r)} .
$$

Since the LDPC codeword consists of $K G F(q)$ symbols, the sphere packing demapper of Figure 2 will process $K$ received sphere-packed symbols, $\left(r_{0}, r_{1}, \ldots, r_{K-1}\right)$, at a time to produce the following $(K \times L)$ soft-metric matrix using Equation (7)

$$
Q=\left[\begin{array}{llll}
Q(0) & Q(1) & \cdots & Q(K-1)
\end{array}\right]^{T},
$$

where,

$Q(k)=\left[\begin{array}{llll}P\left(s_{k}=s^{0} \mid r_{k}\right) & P\left(s_{k}=s^{1} \mid r_{k}\right) & \cdots & P\left(s_{k}=s^{L-1} \mid r_{k}\right)\end{array}\right]$, for $k=0,1, \ldots, K-1$. All the probabilities corresponding to a specific row in $Q$, which correspond to a specific received symbol, should be normalised so that they sum up to unity.

The non-binary rate- 1 decoder of Figure 2 then processes the soft-metric matrix $Q$ of Equation (8) in conjunction with the $a$ priori information, $A_{\text {urc }}$, in order to produce a decoded $a$-poster iori probability matrix, $D_{\text {urc }}$, of size $(K \times L)$ using a standard implementation of the forward-backward recursion based $a$ posteriori probability (APP) algorithm [18]. During the first iteration, $P\left(s^{l}\right), 0 \leq l \leq L-1$, seen in Equation (7) has to be set to $1 / q$, since no a-priori information is yet available from the LDPC decoder. The a-priori knowledge fed into the rate- 1 decoder of Figure 2 is removed from the decoded a-posteriori probability matrix, $D_{\text {urc }}$, using symbol-based element-wise division [14] for the sake of generating the extrinsic probability matrix, $E_{u r c}$, which is appropriately deinterleaved and then fed into the LDPC decoder as the a-priori knowledge, $A_{l d p c}$, as alluded to before. More specifically, the following $(K \times L) a$-priori information matrix is constructed

$$
A_{l d p c}=\left[\begin{array}{llll}
A_{l d p c}(0) & A_{l d p c}(1) & \cdots & A_{l d p c}(K-1)
\end{array}\right]^{T},
$$

where,

$$
A_{l d p c}(k)=\left[\begin{array}{llll}
P\left(s_{k}=s^{0}\right) & P\left(s_{k}=s^{1}\right) & \cdots & P\left(s_{k}=s^{L-1}\right)
\end{array}\right],
$$

and we have

$P\left(s_{k}=s^{l}\right)=\frac{\left(d_{u r c}\right)_{k}^{l}}{\left(a_{u r c}\right)_{k}^{l}}, \quad 0 \leq l \leq L-1, \quad 0 \leq k \leq K-1$, while $\left(d_{u r c}\right)_{k}^{l}$ as well as $\left(a_{u r c}\right)_{k}^{l}$ refer to the elements at the crossover point of the $k^{\text {th }}$ row and $l^{\text {th }}$ column of the matrices $D_{\text {urc }}$ and $A_{u r c}$, respectively. Again, the probabilities corresponding to a specific row of the matrix $A_{l d p c}$ should be normalised, so that the values add up to unity. The LDPC decoder exploits the $a$ priori information, $A_{l d p c}$, for the sake of producing a decoded soft-metric, $D_{l d p c}$. Again, the a-priori information, $A_{l d p c}$, is removed from the decoded a-posteriori probability matrix, $D_{l d p c}$, by symbol-based element-wise division for the sake of generating $E_{l d p c}$, which is passed to the rate-1 decoder of Figure 2 as the $a$ priori knowledge, $A_{u r c}$, for further iterations, until a legitimate codeword is found or the affordable maximum number of iterations has been exhausted.

\section{NON-BINARY EXIT CHART ANALYSIS}

The main objective of employing EXIT charts [13], is to predict the convergence behaviour of the iterative decoder by examining the evolution of the input/output mutual information exchange between the inner and outer decoders in consecutive iterations. Denoting the mutual information between two random variables $X$ and $Y$ as $I(X ; Y)$, the average a-priori information, $I_{A_{u r c}}$, at the input of the inner non-binary rate-1 decoder and the average extrinsic information, $I_{E_{u r c}}$, at the output of the inner nonbinary rate-1 decoder can be defined as [16]

$$
\begin{aligned}
I_{A_{u r c}} & :=\frac{1}{M} \sum_{i=0}^{M-1} I\left(V_{i}^{\pi} ; A_{\text {urc }}(i)\right), \\
I_{E_{u r c}} & :=\frac{1}{M} \sum_{i=0}^{M-1} I\left(V_{i}^{\pi} ; E_{u r c}(i)\right),
\end{aligned}
$$

where $V_{i}^{\pi}$ is an $L$-ary random variable representing the $i^{\text {th }}$ integer symbol, $v_{i}^{\pi}$, at the input of the rate-1 encoder of Figure 1 and $M$ is the total number of legitimate symbols $v_{i}^{\pi}$. Note that $A_{\text {urc }}(i)$ and $E_{\text {urc }}(i)$ are vectors of random variables corresponding to the $i^{t h}$ row of the matrices $A_{u r c}$ and $E_{u r c}$, respectively. The transfer characteristic $T_{u r c}$ of the inner rate- 1 decoder is a function of $I_{A_{u r c}}$ and $E_{b} / N_{0}$ expressed as $I_{E_{u r c}}=T_{u r c}\left(I_{A_{u r c}}, E_{b} / N_{0}\right)$. Similarly, the average a-priori information, $I_{A_{l d p c}}$, at the input of the outer non-binary LDPC decoder and the average extrinsic information, $I_{E_{l d p c}}$, at the output of the outer non-binary LDPC decoder can be defined using Equation (10), where the subscript $u r c$ is replaced with the subscript $l d p c$. The transfer characteristic of the outer non-binary LDPC decoder is given by $I_{E_{l d p c}}=$ $T_{l d p c}\left(I_{A_{l d p c}}\right)$, which does not depend on the $E_{b} / N_{0}$ values. The exchange of extrinsic information in the system schematic of Figure 2 is visualised by plotting the extrinsic information transfer characteristics of the inner non-binary rate-1 decoder and the outer non-binary LDPC decoder in a joint diagram. This diagram is known as the Extrinsic Information Transfer (EXIT) chart [13]. As seen in Figure 2, the outer LDPC decoder's extrinsic output $I_{E_{l d p c}}$ becomes the inner rate- 1 decoder's a-priori input $I_{A_{u r c}}$, which is usually represented on the $x$-axis of the EXIT chart. Similarly, on the $y$-axis of the EXIT chart, the inner rate- 1 decoder's extrinsic output $I_{E_{u r c}}$ becomes the outer LDPC decoder's a-priori input $I_{A_{l d p c}}$, as seen in Figure 2.

The mutual information $I\left(V_{i}^{\pi} ; A_{u r c}(i)\right)$ in Equation (10) can be expressed as $[14,16]$

$$
\begin{aligned}
I\left(V_{i}^{\pi} ; A_{\text {urc }}(i)\right)= & \sum_{v_{i}^{\pi}=0}^{L-1} \int_{\mathrm{a}_{u r c}(i)} p\left(\mathrm{a}_{u r c}(i) \mid v_{i}^{\pi}\right) P\left(v_{i}^{\pi}\right) \\
& \cdot \log _{2}\left(\frac{p\left(\mathrm{a}_{u r c}(i) \mid v_{i}^{\pi}\right)}{p\left(\mathrm{a}_{\text {urc }}(i)\right)}\right) d \mathrm{a}_{u r c}(i),
\end{aligned}
$$

with

$$
p\left(\mathrm{a}_{u r c}(i)\right)=\sum_{v_{i}^{\pi}=0}^{L-1} p\left(\mathrm{a}_{u r c}(i) \mid v_{i}^{\pi}\right) P\left(v_{i}^{\pi}\right),
$$

and the a-priori probabilities $P\left(v_{i}^{\pi}\right)$ for the indices $v_{i}^{\pi}$. The $L$-dimensional integration in (11) can be evaluated numerically, where the pdf $p\left(\mathrm{a}_{u r c}(i) \mid v_{i}^{\pi}\right)$ may be obtained analytically by generating $A_{\text {urc }}(i)$ by observing the $L$-ary received symbol $V_{i}^{\pi}$ arriving over a $\log _{2}(L)$-dimensional Gaussian channel, assuming that the $\log _{2}(L)$ bits corresponding to $V_{i}^{\pi}$ are independent [14]. The term, $I\left(V_{i}^{\pi} ; E_{\text {urc }}(i)\right)$ can also be expressed using Equations (11) and (12), where $\mathrm{a}_{u r c}(i)$ is replaced with $\mathrm{e}_{u r c}(i)$. This requires the determination of the distribution of $p\left(\mathrm{e}_{u r c}(i) \mid v_{i}^{\pi}\right)$ by means 
of Monte Carlo simulations and computing an $L$-dimensional histogram $[13,14]$. However, a more efficient computation of nonbinary EXIT functions was proposed in [15] that requires neither the computation of the $L$-dimensional histogram nor the evaluation of the $L$-dimensional integration in Equation (11). It was shown in [15] that by averaging over a sufficiently large number of length- $K$ blocks, the mutual information $I_{E}$ can be estimated as

$$
I_{E}=-H\left(V_{1}\right)+E\left\{\frac{1}{K} \sum_{i=0}^{K-1} \sum_{l=0}^{L-1} e_{k}^{l}\right\},
$$

where $e_{k}^{l}$ refers to the element at the cross-over point of the $k^{t h}$ row and $l^{\text {th }}$ column of the matrices $E_{u r c}$ or $E_{l d p c}$ and the entropy $H\left(V_{1}\right)$ can be readily determined from the a-priori $L$-ary symbol distributions $P\left(v_{i}\right)$. For example, if we have $P\left(v_{i}=l\right)=1 / L$, for $l=0,1, \ldots, L-1$, (i.e. equiprobable $L$-ary symbols), then arrive at $H\left(V_{1}\right)=-\log _{2}(L)$.

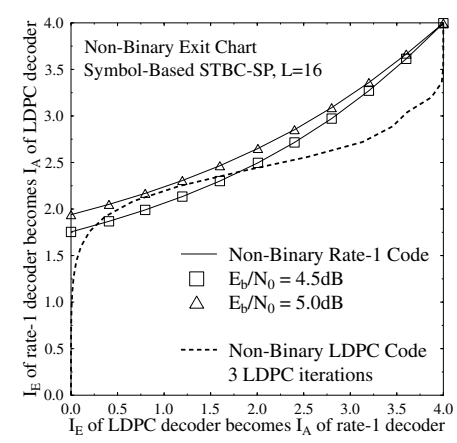

Figure 3: EXIT chart of a non-binary LDPC-coded STBC-SP scheme in combination with the $\frac{1}{2}$-rate outer non-binary LDPC code [9] defined over $G F(16)$ using three internal LDPC iterations and the system parameters outlined in Table 1.

Figure 3 shows the EXIT chart of the turbo-detection aided, non-binary LDPC-coded STBC-SP scheme of Figure 2 in conjunction with the system parameters outlined in Table 1. Ideally, in order for the exchange of extrinsic information between the rate-1 decoder and the outer LDPC decoder of Figure 2 to converge at a specific $E_{b} / N_{0}$ value, the extrinsic transfer curve of the rate-1 decoder recorded at the $E_{b} / N_{0}$ value of interest and the extrinsic transfer characteristic curve of the outer LDPC decoder should only intersect at the point $\left(I_{A}, I_{E}\right)=(4.0,4.0)$. If this condition is satisfied, then a so-called convergence tunnel [13] appears in the EXIT chart. The narrower the tunnel, the closer the system's performance to the channel capacity and hence in the spirit of Shannonian information theory more iterations are required for reaching the $(4.0,4.0)$ point. This is particularly so beyond the cut-off rate of the channel, which is often referred to as the 'practical' channel capacity. Observe in Figure 3 that an open convergence tunnel is taking shape upon increasing the signal-to-noise ratio from $E_{b} / N_{0}=4.5 \mathrm{~dB}$ to $E_{b} / N_{0}=5.0 \mathrm{~dB}$. This implies that according to the predictions of the EXIT chart seen in Figure 3, the iterative decoding process is expected to converge and hence an infinitesimally low $B E R$ may be attained beyond $E_{b} / N_{0}=5.0 \mathrm{~d} B$. However, a high $B E R$ is expected for $E_{b} / N_{0}<4.5 \mathrm{~dB}$ due to the intersection of the extrinsic transfer characteristic curves at a low $I_{E}$ value, as seen in Figure 3. These EXIT chart based convergence predictions are usually verified by the actual iterative decoding curves and trajectory, as it will be discussed in Section 5.

Figure 4 shows the extrinsic information transfer characteristics of the bit-based LDPC benchmarker sphere-packing demapper of in conjunction with $L=16$, when using different mapping schemes between the binary LDPC encoder's output and the STBC

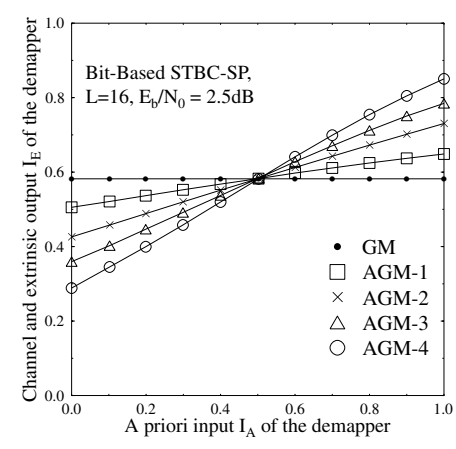

Figure 4: Sphere packing demapper extrinsic information transfer characteristics for Gray mapping $(\mathrm{GM})$ and different bits to sphere-packing symbol anti-Gray mapping (AGM) schemes at $E_{b} / N_{0}=2.5 d B$ for $L=16$, when communicating over a correlated Rayleigh fading channel having a normalised Doppler frequency of $f_{D}=0.1$.

encoder. Accordingly, binary EXIT chart analysis [13] was employed for studying the convergence of the bit-based benchmarker scheme. As was reported in [10], Gray mapping does not always provide an iteration gain upon increasing the mutual information at the input of the demapper. However, using a variety of different Anti-Gray mapping (AGM) schemes [10] results in different extrinsic information transfer characteristics, as illustrated by the different slopes seen in Figure 4. The four different AGM mapping schemes $1-4$ shown in Figure 4 were specifically selected by exhaustive search from all the 16 ! possible mapping schemes available for $L=16$ in order to demonstrate the different extrinsic information transfer characteristics associated with different bitto-symbol mapping schemes.

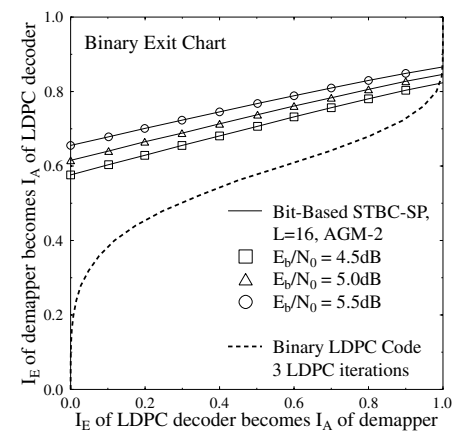

Figure 5: EXIT chart of a turbo-detected binary LDPC-coded STBC-SP scheme employing the Anti-Gray mapping (AGM-2) of Figure 4 in combination with the $\frac{1}{2}$ rate outer binary LDPC code [6] using three internal LDPC iterations and the system parameters outlined in Table 1 .

The EXIT chart of the turbo-detected binary LDPC-coded STBCSP scheme employing the Anti-Gray mapping 2 of Figure 4 in combination with the system parameters outlined in Table 1 is portrayed in Figure 5. According to the figure, a convergence tunnel appears at $E_{b} / N_{0}=5.0 \mathrm{~dB}$, but the extrinsic transfer characteristic curves of the bit-based sphere-packing demapper and the outer binary LDPC decoder intersect at a point infinitesimally close to the $I_{E}=1.0$ line, which indicates that a relatively low $B E R$ may be attained for this particular system arrangement. Nonetheless, there would be a $B E R$ floor preventing the system from achieving an infinitesimally low $B E R$, which is in contrast to the non-binary scheme of Figure 2, where the non-binary EXIT chart seen in Figure 3 demonstrated that convergence to the point $\left(I_{A}, I_{E}\right)=(4.0,4.0)$ was possible. However, the intersection of 
the extrinsic transfer characteristic curves of the bit-based spherepacking demapper and the outer binary LDPC decoder plotted in Figure 5 tends to slide upwards, approaching the $I_{E}=1.0$ line upon gradually increasing the $E_{b} / N_{0}$ values. Hence, it is expected that a reasonably low $B E R$ may be attained even for $E_{b} / N_{0}<5.0 \mathrm{~d} B$, although the $B E R$ reduction versus $E_{b} / N_{0}$ increase would be only gradual, indicating the existence of a nondominant $B E R$ floor, as it will be highlighted in the context of the $B E R$ curve of Figure 9.

\section{RESULTS AND DISCUSSION}

Without loss of generality, we considered a sphere packing modulation scheme associated with $L=16$ using two transmit and a single receiver antenna in order to demonstrate the performance improvements achieved by the proposed system. The specific subset of $L=16$ points selected from the entire set of legitimate constellation points hosted by $D_{4}$ was chosen based on the minimumenergy and optimum minimum Euclidean distance criterion proposed in [17]. All simulation parameters are listed in Table 1.

\begin{tabular}{|l|r|}
\hline Modulation & Sphere Packing with $L=16$ \\
\hline No. of Transmitters & 2 \\
\hline No. of Receivers & 1 \\
\hline Channel & 0.1 \\
\hline $\begin{array}{l}\text { Normalised Doppler } \\
\text { frequency }\end{array}$ & 2.5 \\
\hline $\begin{array}{l}\text { Average LDPC } \\
\text { column weight }\end{array}$ & 0.5 \\
\hline LDPC coding rate & $G F(16)$ \\
\hline $\begin{array}{l}\text { Non-Binary LDPC } \\
\text { decoding field }\end{array}$ & $1 \mathrm{bit} / \mathrm{symbol}$ \\
\hline System throughput & $6000 \mathrm{bits}$ \\
\hline LDPC Coded Blocklength & \\
\hline
\end{tabular}

Table 1: System parameters

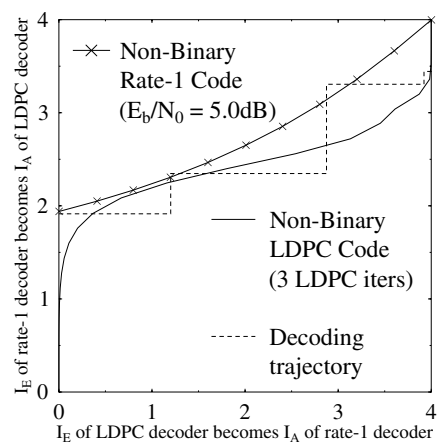

Figure 6: Decoding trajectory of the symbol-based $\frac{1}{2}$-rate LDPC [9] coded STBC$\mathrm{SP}$ scheme in combination with the system parameters outlined in Table 1 and operating at $E_{b} / N_{0}=5.0 \mathrm{~dB}$ after five joint external iterations and three internal LDPC iterations.

Figure 6 illustrates the actual decoding trajectory of the turbodetected non-binary LDPC-coded STBC-SP scheme of Figure 3 at $E_{b} / N_{0}=5.0 \mathrm{~dB}$ after five joint external iterations. The zigzagpath seen in Figure 6 represents the actual extrinsic information transfer between the rate- 1 inner decoder and the outer non-binary LDPC decoder at $E_{b} / N_{0}=5.0 \mathrm{~dB}$. The deviation of the decoding trajectory from the prediction of the EXIT chart in Figure 3 is due to the fact that the assumption of having $\log _{2}(L)$ independent bits corresponding to each index $V_{i}$ is only approximately valid. This assumption was exploited when creating $A_{\text {urc }}(i)$ for the sake of generating the appropriate a-priori information $I_{A}$ value to characterise the extrinsic information transfer characteristics of the constituent decoders. However, the $B E R$ curves of the

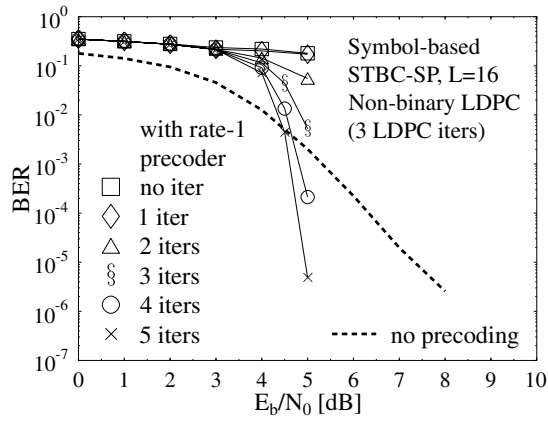

Figure 7: Performance of symbol-based $\frac{1}{2}$-rate LDPC [9] coded STBC-SP schemes in combination with the system parameters outlined in Table 1 after five joint external iterations and three internal LDPC iterations.

scheme characterised in Figure 3, which are shown in Figure 7, match the predictions of the EXIT chart quite accurately in Figure 3 , since the predicted turbo cliff at $E_{b} / N_{0}=5.0 \mathrm{~dB}$ and the associated infinitesimally low $B E R$ become quite evident.

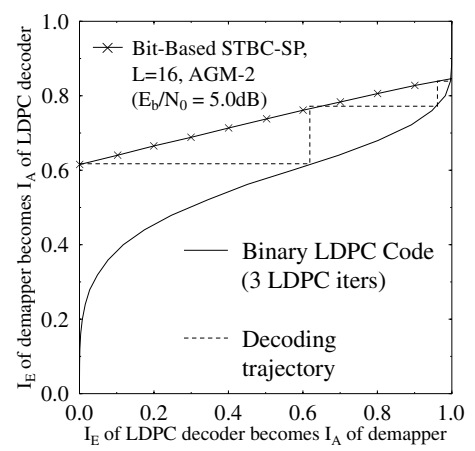

Figure 8: Decoding trajectory of a bit-based LDPC-coded STBC-SP scheme in conjunction with Anti-Gray Mapping (AGM-2) and in combination with the system parameters outlined in Table 1 after 10 joint external iterations and three internal LDPC iterations.

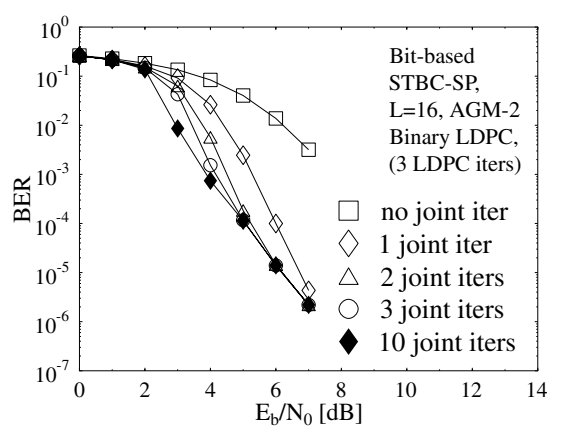

Figure 9: Performance of a bit-based LDPC-coded STBC-SP scheme in conjunction with Anti-Gray Mapping (AGM-2) and in combination with the system parameters outlined in Table 1 after 10 joint external iterations and three internal LDPC iterations.

Figure 8 illustrates the decoding trajectory of the $\frac{1}{2}$-rate turbodetected binary LDPC [6] coded STBC-SP scheme of Figure 5 operating at $E_{b} / N_{0}=5.0 \mathrm{~dB}$ after 10 joint external iterations and three internal LDPC iterations. Observe in Figure 8 that three joint external iterations was sufficient for reaching the intersection point. Figure 9 shows the $B E R$ performance of the bit-based LDPC-coded STBC-SP scheme of Figure 5. The gentle slope of the $B E R$ curve seen in Figure 9 matches the predictions of the 
EXIT chart seen in Figure 5. Additionally, note that the $B E R$ curves after three and 10 joint external iterations become almost identical for $E_{b} / N_{0}>4.0 \mathrm{~dB}$, since having three joint external iterations is sufficiently high for achieving the highest possible iteration gain, as observed for the decoding trajectory of Figure 9.

Figure 10 compares the attainable performance of both the symbol-based non-binary LDPC-coded STBC-SP scheme and the bitbased LDPC-coded STBC-SP scheme in conjunction with AntiGray Mapping (AGM-2) when using the system parameters outlined in Table 1 after five joint external iterations and three internal LDPC iterations against an identical-throughput $1 \mathrm{bit} / \mathrm{symbol}$ (BPS) uncoded STBC-SP scheme [5] using $L=4$ and against Alamouti's conventional $G_{2}$-BPSK scheme [3]. Observe the difference in Figure 10 between the slope of the symbol-based and bit-based schemes, which was as expected for the EXIT chart results. Additionally, a useful performance improvement was obtained, when performing iterative decoding between the rate- 1 inner and the non-binary outer LDPC decoders. Explicitly, Figure 10 demonstrates that a coding advantage of about $19.2 \mathrm{~dB}$ was achieved at a BER of $10^{-5}$ after five joint external iterations by the symbol-based non-binary LDPC-coded STBC-SP scheme when, using three internal LDPC iterations over both the uncoded STBC-SP [5] and the conventional orthogonal STBC design based schemes $[3,4]$ for transmission over the correlated Rayleigh fading channel having $f_{D}=0.1$. Additionally, a coding advantage of approximately $1.3 d B$ was attained over the 1BPS-throughput bitbased LDPC-coded STBC-SP scheme in conjunction with AntiGray Mapping (AGM-2), when using the system parameters of Table 1.

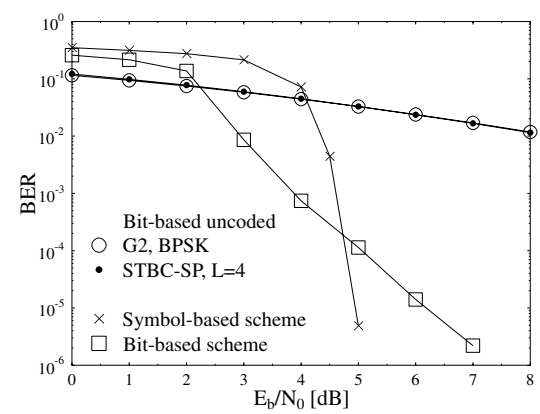

Figure 10: Performance comparison of the symbol-based $\frac{1}{2}$-rate non-binary LDPC [9] coded STBC-SP scheme and the bit-based $\frac{1}{2}$-rate binary LDPC [6] coded STBC-SP scheme in conjunction with the Anti-Gray Mapping (AGM-2) and in combination with the system parameters outlined in Table 1 after five joint external iterations and three internal LDPC iterations against an identical-throughput 1 bit/symbol (BPS) uncoded STBC-SP scheme using $L=4$ and against Alamouti's conventional $G_{2}$-BPSK scheme.

\section{CONCLUSION}

In this paper we proposed a novel symbol-based iterative system that exploits the advantages of non-binary LDPC codes [9], those of the rate-1 inner codes of [11] as well as those of the STBCSP scheme of [5]. Our investigations demonstrated that significant performance improvements may be achieved by the proposed scheme over established orthogonal STBC designs, constituted by the STBC-SP scheme of [5] as well as over an equivalent bit-based LDPC-coded STBC-SP scheme. Subsequently, non-binary EXIT charts were used to study the convergence of the proposed symbolbased scheme. By contrast, binary EXIT charts were used for designing the bit-based binary LDPC-coded STBC-SP scheme's optimum bit-to-symbol mapping schemes, which facilitated convergence at the lowest possible $E_{b} / N_{0}$ values. Several STBCSP mapping schemes covering a wide range of extrinsic transfer characteristics were investigated. Our analysis demonstrated that useful performance improvements can be obtained, when performing iterative decoding between the rate- 1 inner and the outer non-binary LDPC decoders over the uncoded STBC-SP arrangement [5], as well as over the conventional orthogonal STBC design based schemes $[3,4]$ and the bit-based LDPC-coded STBCSP scheme

\section{REFERENCES}

[1] L. Hanzo, T. H. Liew, and B. L. Yeap, Turbo Coding, Turbo Equalisation and Space-Time Coding: for Transmission over Fading Channels. Chichester, England: John Wiley and Sons Ltd and IEEE Press, NY, USA, 2002.

[2] B. M. Hochwald, G. Gaire, B. Hassibi, and E. T. L. Marzetta, "Special issue on space-time transmission, reception, coding and signal processing," IEEE Transactions on Information Theory, vol. 49, pp. 2329-2806, Oct 2003.

[3] S. Alamouti, "A simple transmit diversity technique for wireless communications," IEEE Journal on Selected Areas in Communications, vol. 16, no. 8, pp. 1451-1458, 1998.

[4] V. Tarokh, H. Jafarkhani, and A. Calderbank, "Space-time block codes from orthogonal designs," IEEE Transactions on Information Theory, vol. 45, pp. 1456-1467, Jul 1999.

[5] W. Su, Z. Safar, and K. J. R. Liu, "Space-time signal design for timecorrelated Rayleigh fading channels," in IEEE International Conference on Communications, vol. 5, (Anchorage, Alaska), pp. 31753179,2003

[6] R. Gallager, "Low density parity check codes," IEEE Transactions on Information Theory, vol. 8, pp. 21-28, Jan. 1962.

[7] T. Richardson and R. Urbanke, "The renaissance of Gallager's low-density parity-check codes," IEEE Communications Magazine, vol. 41, pp. 126-131, Aug 2003.

[8] C. Berrou, A. Glavieux, and P. Thitimajshima, "Near Shannon limit error-correcting coding and decoding: Turbo codes," Proceedings of the IEEE International Confrence on Communications, pp. 10641070, May 1993.

[9] M. C. Davey and D. J. C. MacKay, "Low density parity check codes over GF(q)," IEEE Communications Letters, vol. 2, pp. 165-167, June 1998.

[10] S. ten Brink, J. Speidel, and R.-H. Yan, "Iterative demappping and decoding for multilevel modulation," in IEEE Global Telecommunications Conference, vol. 1, (Sydney, Australia), pp. 579-584, 8-12 Nov 1998

[11] D. Divsalar, S. Dolinar, and F. Pollara, "Serial concatenated trellis coded modulation with rate-1 inner code," in IEEE Global Telecommunications Conference, vol. 2, pp. 777-782, 27 Nov-1 Dec 2000.

[12] L. Lifang, D. Divsalar, and S. Dolinar, "Iterative demodulation, demapping, and decoding of coded non-square QAM," in IEEE Transactions on Communications, vol. 53, pp. 16-19, Jan 2005.

[13] S. ten Brink, "Designing iterative decoding schemes with the extrinsic information transfer chart," AEÜ International Journal of Electronics and Communications, vol. 54, pp. 389-398, Nov 2000.

[14] A. Grant, "Convergence of non-binary iterative decoding," IEEE Global Telecommunications Conference, vol. 2, pp. 1058-1062, Nov 2001.

[15] J. Kliewer, S. X. Ng, and L. Hanzo, "On the computation of exit characteristics for symbol-based iterative decoding," to appear in 4th International Symposium on Turbo Codes in connection with 6th International ITG-Conference on Source and Channel Coding, 2006.

[16] I. Land, P. Hoeher, and S. Gligorevic, "Computation of symbol-wise mutual information in transmission systems with $\log$ APP decoders and application to EXIT charts," in Proceedings of the International ITG Conference on Source and Channel Coding (SCC), (Erlangen, Germany), pp. 195-202, Jan 2004.

[17] O. Alamri, B. L. Yeap, and L. Hanzo, "Turbo detection of channelcoded space-time signals using sphere packing modulation," in IEEE Vehicular Technology Conference, vol. 4, (Los Angeles, USA), pp. 2498-2502, Sep 2004.

[18] L. Bahl, J. Cocke, F. Jelinek, and J. Raviv, "Optimal decoding of linear codes for minimizing symbol error rate," in IEEE Transactions on Information Theory, vol. 20, pp. 284-287, March 1974. 